\title{
STRATÉGIE DU DIAGNOSTIC EN ENDOSCOPIE DIGESTIVE
}

L'endoscopie digestive passe en haute résolution avec le vidéo-endoscope et le traitement électronique de l'image. Avec la miniaturisation du capteur, le Charge Coupled Device (CCD) apporte, sur une surface réduite, plus de pixels pour un même diamètre de l'endoscope; ce qui implique l'évolution du processeur et l'utilisation d'un moniteur spécifique tandis que s'annonce la mutation vers la télévision en haute définition (HDTV). L'image réfléchie par la muqueuse éclairée en lumière blanche peut être traitée électroniquement en rehaussant les détails de la surface (structure enhancement) ou la couleur de l'hémoglobine contenue dans le tissu (Hemoglobin Index). On peut aussi créer un effet de chromoscopie avec des images électroniques de synthèse combinées pour des longueurs d'ondes choisies dans le rouge, le vert et le bleu (système FICE de Fujinon). Si des filtres sont interposés sur la lumière incidente éclairant la muqueuse pour créer des faisceaux étroits dans le rouge, le vert, le bleu (imagerie en bandes étroites), les images réfléchies correspondent, avant leur combinaison, à la surface de la muqueuse, sa partie moyenne et sa partie profonde. La technique NBI d'Olympus augmente le contraste du réseau vasculaire superficiel, dessiné en bleu-vert sous la surface de la muqueuse, et renforce le relief des cryptes et crêtes épithéliales; elle peut être couplée à l'endoscopie en magnification : le zoom optique est utilisé aux grossissements $\times 60$ à $\times 80 ;$ l'objectif «macro», plus maniable, grossit moins. Le zoom électronique permet un agrandissement utile des détails surtout en HDTV. L'endocytoscopie et la microscopie explorent à l'échelle des cellules, mais sont encore des techniques expérimentales.

En soi, le progrès de l'image est évident, surtout pour les lésions planes digestives, mais une plus belle image ne signifie pas toujours un meilleur diagnostic. La distinction entre variations de la normale, anomalies sans valeurs pathologiques ou franchement pathologiques, devient subtile. L'attitude simpliste, «je résèque tout ce qui me paraît anormal et $\mathrm{j}$ 'attends l'histologie» n'est plus de mise. Le diagnostic endo- scopique exige une stratégie en plusieurs étapes. Le contrôle de qualité s'applique dès la préparation pour l'examen : la muqueuse doit être propre et sèche car tout résidu solide ou liquide peut masquer des lésions planes en coloscopie. L'examen de la muqueuse gastrique est plus précis après administration de Siméthicone ou de Pronase.

Le premier temps de l'endoscopie est la détection de «cibles» en scrutant toute la surface de la muqueuse. L'image associée aux lésions polypoïdes ou ulcérées est ancrée dans la mémoire visuelle des endoscopistes occidentaux; en revanche, celle des lésions planes, légèrement surélevées ou déprimées, ne l'est pas : simple changement de couleur sur une zone ou discontinuité dans le réseau vasculaire superficiel.

Si l'opérateur a repéré une "cible", le deuxième temps est la caractérisation en tant que variante de la normale, lésion non néoplasique ou néoplasique. 1 - La chromoscopie (indigo carmin), focalisée sur la «cible», permet l'analyse morphologique des surélévations ou des dépressions. 2 - Le NBI est la technique spécifique pour l'examen des petits vaisseaux superficiels : des stades distincts sont décrits pour l'inflammation, la néoplasie non invasive, le cancer. 3 - Si les anomalies vasculaires évoquent la néoplasie, l'examen de la surface (Pit pattern) avec zoom optique et chromoscopie (indigo carmin) peut prédire le caractère invasif ou non invasif de la lésion.

C'est alors le troisième temps de décision thérapeutique nuancée : pas de traitement pour un aspect normal, inflammatoire ou un petit polype hyperplasique, résection ou simple surveillance pour une lésion en dysplasie bas grade de quelques millimètres de diamètre, résection endoscopique pour une lésion en dysplasie haut grade d'aspect non invasif, indication directe de la chirurgie si l'invasion profonde de la sous-muqueuse est suspectée.

René LAMBERT

Septembre 2006 